(C2007 IEEE. Personal use of this material is permitted. However, permission to reprint/republish this material for advertising or promotional purposes or for creating new collective works for resale or redistribution to servers or lists, or to reuse any copyrighted component of this work in other works must be obtained from the IEEE. 


\title{
FIR Variable Digital Filter With Signed Power-of-Two Coefficients
}

\author{
Hai Huyen Dam, Antonio Cantoni, Fellow, IEEE, Kok Lay Teo, Senior Member, IEEE, and \\ Sven Nordholm, Senior Member, IEEE
}

\begin{abstract}
Variable digital filters (VDFs) are useful for various signal processing and communication applications where the frequency characteristics, such as fractional delays and cutoff frequencies, can be varied online. In this paper, we investigate the design of VDFs with discrete coefficients as a means of achieving low complexity and efficient hardware implementation. The filter coefficients are expressed as the sum of signed power-of-two terms with a restriction on the total number of power-of-two for the filter coefficients. An efficient design procedure is proposed that includes an improved method for handling the quantization of the VDF coefficients for both the min-max and the least-square criteria leading to an optimum quantized solution. For the least-square criterion, a reduced search region around the optimum quantized solution is further constructed and the branch and bound method in conjunction with an efficient branch cutting scheme is presented to search for an optimum solution in this reduced region.
\end{abstract}

Index Terms-Finite-impulse response (FIR) variable digital filter (VDF), least-square criteria, min-max, optimization, signed power-of-two (SPT) coefficients.

\section{INTRODUCTION}

$\mathbf{V}$ ARIABLE digital filters (VDFs) are digital filters with controllable spectral characteristics such as variable cutoff frequency, adjustable passband width and controllable fractional delay [1]-[3]. These spectral characteristics can be varied online. VDFs have many applications in different areas of signal processing and communications. Examples include arbitrary sample rate changers, digital synchronizers and other applications involving online tuning of frequency characteristics [4], [5]. Fractional delay digital filters have various important applications including timing adjustment for digital receivers [4].

In this paper, we investigate the design of the Farrow-based VDF [6] with coefficients expressed as the sum of signed power-of-two (SPT) terms. These coefficients are attractive

Manuscript received September 29, 2005; revised October 16, 2006. This work was supported by the Australia Research Council under Grant DP0451111. This paper was recommended by Y. Lian.

H. H. Dam is with Western Australian Telecommunications Research Institute (WATRI), Crawley, WA 6009, Australia, and also with the Department of Mathematics and Statistics, Curtin University of Technology, Perth, WA 6845, Australia (e-mail: dam@watri.org.au).

A. Cantoni is with Western Australian Telecommunications Research Institute (WATRI), The University of Western Australia, Perth, WA 6009, Australia (e-mail: cantoni@watri.org.au)

K. L. Teo is with Department of Mathematics and Statistics, Curtin University of Technology, Perth, WA 6845, Australia (e-mail: K.L.Teo@curtin.edu.au).

S. Nordholm is with Western Australian Telecommunications Research Institute (WATRI), Curtin University of Technology, Perth, WA 6845, Australia (e-mail: sven@watri.org.au)

Digital Object Identifier 10.1109/TCSI.2007.897775 for hardware implementation since multiplications can be efficiently implemented by using shifters or adders. This will result in low cost, low complexity and ease in implementations for the filters as well as high speed and high yield. These advantages have been explored and consequently incorporated in various cases [7]-[11].

In [12]-[14], the design of VDF with the sum of SPT coefficients and the min-max criterion is investigated. Here, we examine a general design of the VDF with both the min-max and the least-square criteria in conjunction with a restriction on the total number of power-of-two of the filter coefficients. This restriction allows the control on the total SPT for the filter coefficients, thereby determining the complexity of the filter implementation. In other words, the design optimizes the number of power-of-two required to be distributed for each VDF coefficients, given a restriction on the total number of SPT terms for the filter [15].

In [12] and [13], a random search algorithm is employed for the design of the VDF with the sum of SPT coefficients and the min-max criterion. Here, we develop efficient design schemes for the VDFs with the sum of SPT coefficients for both the min-max and the least-square criteria. For the min-max criterion, a two-step procedure is proposed which includes a quantization scheme to efficiently distribute the SPT to the filter coefficients, leading to an optimum quantized solution. For the least-square criterion, a three-step procedure is developed which incorporates a global branch and bound algorithm to search further in a reduced region around the optimum quantized solution. The reason of incorporating another step for this criterion instead of the min-max criterion is that the bounds for the branch and bound algorithm can be obtained with low complexity especially when the VDFs are designed with controllable characteristics. The performance of the scheme is compared with the solution obtained in [13].

The paper is organized as follows. The VDF and the desired frequency response are given in Section II. The VDF with coefficients expressed as the sum of SPT terms is presented in Section III. A two-step scheme which includes the quantization procedure and optimization with respect to the scaling factor for both the min-max and the least-square criteria is developed in Section IV. For the least-square criterion, the designed problem is further formulated as a mixed integer programming problem in Section V. A reduced search region is constructed and the branch and bound algorithm with an efficient branch cutting scheme is presented for solving the problem. Design examples solved by using the proposed procedure are shown in Section VI. Finally, conclusions are given in Section VII. 


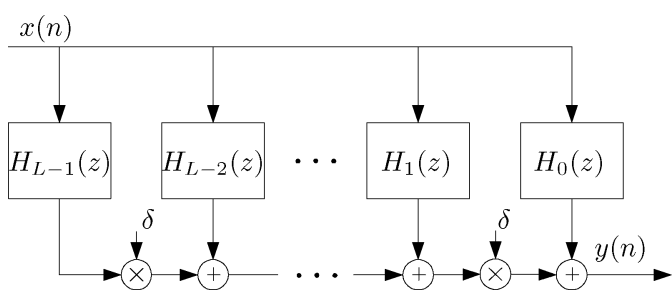

Fig. 1. VDF-Farrow structure.

\section{VDF AND THE DESIRED FREQUENCY RESPONSE}

In a VDF design problem, the objective is to achieve a design response $H_{d}(z, \delta)$ that is a function of a control or tuning parameter $\delta$ defined to lie in some range $\Delta=\left[\delta_{\min }, \delta_{\max }\right]$. For example, the tuning parameter may control the cutoff frequency of the filter or the delay of the filter in some specified bandwidth. The formulation will also take into consideration of meeting additional requirements of the filter in the frequency response.

In this paper, we are concerned with the design of VDF shown in Fig. 1. The structure includes $L$ FIR sub-filters $H_{l}(z), 0 \leq$ $l \leq L-1$. The tuning parameter $\delta$ is the input to $L-1$ multipliers. The structure referred as the Farrow structure is described in [6]. The frequency response of a sub-filter $H_{l}(z)$ with the corresponding impulse response $h_{l}(n)$ is given as

$$
H_{l}(z)=\sum_{n=0}^{N-1} h_{l}(n) z^{-n}=\mathbf{h}_{l}^{T} \phi(z)
$$

where $\mathbf{h}_{l}=\left[h_{l}(0), \ldots, h_{l}(N-1)\right]^{T}$ and $\phi(z)=$ $\left[z^{0}, \ldots, z^{-(N-1)}\right]^{T}$. The notation $[\cdot]^{T}$ denotes the transpose of a vector and $N$ is the length of the FIR sub-filters.

The frequency response of the VDF can be expressed as

$$
\begin{aligned}
H(z, \delta) & =\sum_{l=0}^{L-1} H_{l}(z) \delta^{l} \\
& =\sum_{n=0}^{N-1} \sum_{l=0}^{L-1} h_{l}(n) \delta^{l} z^{-n} \quad \forall \delta \in \Delta .
\end{aligned}
$$

Let $\mathbf{h}$ denote the $N L \times 1$ vector of the VDF coefficients

$$
\mathbf{h}=\left[\mathbf{h}_{0}^{T}, \ldots, \mathbf{h}_{L-1}^{T}\right]^{T}(3)
$$

and $\mathbf{s}(\omega, \delta)$ be an $N L \times 1$ complex vector $\mathbf{s}(\omega, \delta)=$ $\left[\phi^{T}\left(e^{j \omega}\right) \delta^{0}, \ldots, \phi^{T}\left(e^{j \omega}\right) \delta^{L-1}\right]^{T}$. Then, the frequency response of the VDF in (2) can be rewritten in the following form:

$$
H\left(e^{j \omega}, \delta\right)=\mathbf{h}^{T} \mathbf{s}(\omega, \delta) .
$$

The desired frequency response for the VDF is specified by where $\mathcal{P}(\delta)$ and $\mathcal{S}(\delta)$ are, respectively, the passband and stopband regions, whilst $\tau(\delta)$ is the desired group delay. In general, the VDFs are designed with: (1) variable fractional delay; or (2) variable cutoff frequency. For a variable fractional delay filter, the desired group delay $\tau(\delta)$ is tunable via $\delta$ and is given by

$$
\tau(\delta)=\tau_{d}+\beta_{\tau}(\delta)
$$

where $\beta_{\tau}(\delta)$ is a continuous function with respect to $\delta$. For a variable cutoff frequency filter, the passband and stopband regions, $\mathcal{P}(\delta)$ and $\mathcal{S}(\delta)$, vary depending on $\delta$.

\section{VDF WITH COEFFICIENTS EXPRESSED AS THE SUM OF SPT TERMS}

In this section, we express the VDF coefficients in terms of the sum of SPT. Each sum of SPT is restricted by a maximum number of allowable bits, denoted by a positive integer $B$, and is given as $\sum_{k=1}^{c} \eta_{k} 2^{-\nu_{k}}$ where $c \geq 0$ represents the number of SPT terms, $\eta_{k}$ is a binary value and $\nu_{k}$ is an integer in the set $\{1, \ldots, B\}$. Thus, the range for each sum of SPT is in $[-1+$ $\left.2^{-B}, 1-2^{-B}\right]$. For the filter to satisfy a general specification, a common scaling factor $\gamma$ is introduced and incorporated to scale the sum of SPT filter coefficients. Thus, the coefficient vector $\mathbf{h}$ of the VDF includes a sum of SPT coefficient vector, denoted by $\mathrm{g}$, and a scaling factor $\gamma$. In other words, the VDF coefficient vector can be given as

$$
\mathbf{h}=\gamma \mathbf{g}
$$

where each coefficient $g_{l}(n)$ of $\mathbf{g}$ can be expressed as

$$
\begin{aligned}
g_{l}(n) & =\sum_{k=1}^{c_{l, n}} \eta_{l, n, k} 2^{-\nu_{l, n, k}} \\
c_{l, n} & \geq 0 \quad \forall 0 \leq l \leq L-1,0 \leq n \leq N-1
\end{aligned}
$$

with $\eta_{l, n, k} \in\{-1,1\}$ and $\nu_{l, n, k} \in\{1, \ldots, B\}$. Note that $c_{l, n}=0$ implies that $g_{l}(n)=0$. Since $B$ is the total number of allowable bits for each coefficient, we have

$$
c_{l, n} \leq B \quad \forall l \text { and } n .
$$

The frequency response of the VDF then becomes

$$
H\left(e^{j \omega}, \delta\right)=\gamma G\left(e^{j \omega}, \delta\right)
$$

where $G\left(e^{j \omega}, \delta\right)$ is the frequency response of the discrete filter corresponding to the coefficient vector $\mathrm{g}$.

The total number of SPT terms for the VDF coefficients is restricted to a positive number $M$ according to

$$
\sum_{l=0}^{L-1} \sum_{n=0}^{N-1} c_{l, n} \leq M .
$$


For convenience of notation, we now define a region $\mathcal{G}$ of $\mathrm{g}$ where each coefficient is discrete and can be represented as the sum of SPT terms with a restriction of $B$ bits

$$
\begin{aligned}
\mathcal{G}= & \left\{\mathrm{g}: g_{l}(n)=\sum_{k=1}^{c_{l, n}} \eta_{l, n, k} 2^{-\nu_{l, n, k}}\right. \\
& \forall 0 \leq l \leq L-1,0 \leq n \leq N-1, \\
& \left.c_{l, n} \leq B, \eta_{l, n, k} \in\{-1,1\} \text { and } \nu_{l, n, k} \in\{1, \ldots, B\}\right\} .
\end{aligned}
$$

In the following, a two-step quantization scheme for the leastsquare and min-max criteria will be developed.

\section{QUANTIZATION PROCEDURE AND OPTIMIZATION WiTH RESPECT TO THE SCALING FACTOR}

From [12], [13], the least-square infinite precision solution is employed to obtain the quantized solution with total number of SPT terms restricted to a positive number $M$. We now formulate the least-square criterion for the design of VDF with infinite precision coefficients. The integral squared deviation between the frequency response $H\left(e^{j \omega}, \delta\right)$ and the desired response $H_{d}\left(e^{j \omega}, \delta\right)$ over $\delta \in \Delta$ is given by

$$
E(\mathbf{h})=\int_{\Delta} \int_{\Omega(\delta)} W^{2}(\omega, \delta)\left|H\left(e^{j \omega}, \delta\right)-H_{d}\left(e^{j \omega}, \delta\right)\right|^{2} d \omega d \delta
$$

where $\Omega(\delta)$ is the range of the frequency $\omega, \Omega(\delta)=\mathcal{P}(\delta) \cup$ $\mathcal{S}(\delta)$, and $W(\omega, \delta)$ is the weighting function depending on $\omega$ and $\delta$. Since the coefficient vector $\mathbf{h}$ of the VDF is real, the cost function (10) can be reduced to the following quadratic function [17]

$$
E(\mathbf{h})=\mathbf{h}^{T} \mathbf{Q h}+\mathbf{p}^{T} \mathbf{h}+\alpha
$$

where

$$
\begin{aligned}
& \mathbf{Q}=\Re\left\{\int_{\Delta} \int_{\Omega(\delta)} W^{2}(\omega, \delta) \mathbf{s}(\omega, \delta) \mathbf{s}^{H}(\omega, \delta) d \omega d \delta\right\} \\
& \mathbf{p}=-2 \Re\left\{\int_{\Delta} \int_{\Omega(\delta)} W^{2}(\omega, \delta) \mathbf{s}(\omega, \delta) H_{d}^{*}\left(e^{j \omega}, \delta\right) d \omega d \delta\right\}
\end{aligned}
$$

and $\alpha$ is a constant scalar given by $\alpha=\int_{\Delta} \int_{\Omega(\delta)} W^{2}(\omega, \delta)\left|H_{d}\left(e^{j \omega}, \delta\right)\right|^{2} d \omega d \delta \quad$ with $(\cdot)^{H}$ and $\Re\{\cdot\}$ denoting, respectively, the Hermitian transpose and the real component of a complex vector.

The infinite precision solution $\mathbf{h}_{L S}$ to the least-squares design problem which minimizes the quadratic cost function (11) is given as

$$
\mathbf{h}_{L S}=-\frac{1}{2} \mathbf{Q}^{-1} \mathbf{p}
$$

For a scaling factor $\gamma$, it follows from (7) that the integral squared error for the VDF with discrete coefficients $g$ can be given as

$E(\mathbf{g}, \gamma)=\int_{\Delta} \int_{\Omega(\delta)} W^{2}(\omega, \delta)\left|\gamma G\left(e^{j \omega}, \delta\right)-H_{d}\left(e^{j \omega}, \delta\right)\right|^{2} d \omega d \delta$
Another approach to obtain the discrete filters is to scale the desired frequency response as in [11]. This scaling factor is then required to be incorporated into the cost function. For a leastsquare criterion, this incorporation results in a nonconvex cost function for the infinite precision solution filter and hence the minimum for the cost function is not readily obtained. Hence, in this paper, we focus on the case in (13).

From (11), (13) is reduced to

$$
E(\mathbf{g}, \gamma)=\gamma^{2} \mathbf{g}^{T} \mathbf{Q g}+\gamma \mathbf{p}^{T} \mathbf{g}+\alpha
$$

The infinite precision, that is unconstrained solution to the leastsquare problem

$$
\min _{\mathbf{g} \in \mathcal{R}^{N L}} \gamma^{2} \mathbf{g}^{T} \mathbf{Q g}+\gamma \mathbf{p}^{T} \mathbf{g}+\alpha
$$

is given by

$$
\mathrm{g}_{L S, \gamma}=\frac{\mathbf{h}_{L S}}{\gamma}, \gamma \neq 0
$$

where $\mathbf{h}_{L S}$ is given in (12).

As the first step, we develop a quantization procedure for the VDF coefficients given a scaling factor $\gamma$.

\section{A. Quantization Procedure for a Fixed Scaling Factor $\gamma$}

We extend the quantization method proposed in [15] to the coefficients of the VDF structure. For a given $\gamma$, the quantized solution $g_{\gamma}$ that lies in the region defined by (9) and satisfies the constraint (8) can be obtained by quantizing the coefficients of the infinite precision solution $\mathrm{g}_{L S, \gamma}$ to $B$ bits. The procedure for obtaining this quantized solution is given as follows.

1) Procedure 4.1: Obtain quantized solution $\mathrm{g}_{\gamma}$ with $\max -$ imum $B$ bits from the infinite precision solution $\mathbf{g}_{L S, \gamma}$ given an upper bound $M$ on the total number of SPT terms.

Step 1) Initialize $\mathbf{g}_{\gamma}=\mathbf{0}_{N L, 1}$, where $\mathbf{0}_{N L, 1}$ denotes an $N L \times 1$ zero vector

$$
g_{\gamma, l}(n)=0 \quad \forall 0 \leq l \leq L-1 \text { and } 0 \leq n \leq N-1
$$

Set an intermediate vector $\hat{\mathbf{g}}_{\gamma}$ as $\hat{\mathbf{g}}_{\gamma}=\mathbf{g}_{L S, \gamma}$, and $k=1$.

Step 2) Search for the indexes $l_{1}, 0 \leq l_{1} \leq L-1$, and $n_{1}$ $0 \leq n_{1} \leq N-1$, corresponding to the coefficient in $\hat{\mathrm{g}}_{\gamma}$ with the maximum absolute value

$$
\left|\hat{g}_{\gamma, l_{1}}\left(n_{1}\right)\right|=\max _{0 \leq l \leq L-1} \max _{0 \leq n \leq N-1}\left|\hat{g}_{\gamma, l}(n)\right| \text {. }
$$

We have the following two cases.

- If the absolute value of $\hat{g}_{\gamma, l_{1}}\left(n_{1}\right)$ is less than $2^{-B-1}$, then from (17) the absolute values of all the coefficients in $\hat{\mathrm{g}}_{\gamma}$ are less than $2^{-B-1}$. Go to Step 4.

- Otherwise, $\left|\hat{g}_{\gamma, l_{1}}\left(n_{1}\right)\right| \geq 2^{-B-1}$. The $k^{t h}$ SPT term will be allocated to the $\left(l_{1}, n_{1}\right)^{t h}$ position of $\mathbf{g}_{\gamma}$. We now search for a SPT term that is closest to $\hat{g}_{\gamma, l_{1}}\left(n_{1}\right)$. Denote by $\mathcal{B}$ the set of all possible SPT terms

$$
\mathcal{B}=\left\{2^{-1}, \ldots, 2^{-B},-2^{-1}, \ldots,-2^{-B}\right\} .
$$


Search for a number $\zeta \in \mathcal{B}$ that is closest to $\hat{g}_{\gamma, l_{1}}\left(n_{1}\right)$

$\left|\hat{g}_{\gamma, l_{1}}\left(n_{1}\right)-\zeta\right|=\min _{\zeta_{1} \in \mathcal{B}}\left|\hat{g}_{\gamma, l_{1}}\left(n_{1}\right)-\zeta_{1}\right|$.

and go to Step 3 .

Step 3) Update the quantized vector $\mathbf{g}_{\gamma}$ by adding the SPT term $\zeta$ to the coefficient $g_{\gamma, l_{1}}\left(n_{1}\right)$ at the position $l=l_{1}$ and $n=n_{1}$

$$
g_{\gamma, l_{1}}\left(n_{1}\right)=g_{\gamma, l_{1}}\left(n_{1}\right)+\zeta .
$$

Furthermore, update the vector $\hat{\mathbf{g}}_{\gamma}$ by subtracting $\zeta$ from $\hat{g}_{\gamma, l_{1}}\left(n_{1}\right)$

$$
\hat{g}_{\gamma, l_{1}}\left(n_{1}\right)=\hat{g}_{\gamma, l_{1}}\left(n_{1}\right)-\zeta .
$$

If the number of iterations $k$ is less than the number of allowable SPT terms in the filter coefficients, $k<$ $M$, then set $k=k+1$ and return to Step 2. Otherwise, go to Step 4.

Step 4) Stop the procedure. The vector $g_{\gamma}$ is the quantized solution corresponding to the scaling factor $\gamma$, satisfying the SPT constraint.

Since each VDF coefficient is not restricted to a fixed number of SPT terms, there is a greater degree of freedom in distributing the SPT terms to the appropriate filter coefficients given a fixed allowable number of SPT.

\section{B. Search Region for $\gamma$}

We now establish the search region for $\gamma$. Since $\left(\mathrm{g}_{\gamma}, \gamma\right)$ and $\left(-\mathrm{g}_{\gamma},-\gamma\right)$ result in filters with the same integral squared error and the same total number of SPT terms, we only need to search for $\gamma$ in $(0, \infty)$.

For a fixed number of bits $B$, let us define $\gamma_{q}$ as the value of the scaling factor in which the maximum absolute value of $\mathbf{g}_{L S, \gamma_{q}}$ equals to $1-2^{-B}$

$$
\gamma_{q}=\max _{0 \leq l \leq L-1} \max _{0 \leq n \leq N-1} \frac{\left|h_{L S, l}(n)\right|}{1-2^{-B}} .
$$

To restrict the range of $\gamma$, note the following.

- If $\gamma \geq 4 \gamma_{q}$ then the maximum absolute value of all the coefficients of $\mathbf{g}_{L S, \gamma}$ is shown in (23) at the bottom of the page. Thus, the number of bits required is smaller than $B-1$.

- If $\gamma<\gamma_{q}$ then we have

$\max \left|\mathbf{g}_{L S, \gamma}\right|=\max _{0 \leq l \leq L-1} \max _{0 \leq n \leq N-1} \frac{\left|h_{L S, l}(n)\right|}{\gamma}>1-2^{-B}$.

Hence, overflow occurs. When $\gamma$ is much smaller than $\gamma_{q}$, this will result in a large truncation for the quantized solution, leading to a large quantization error. In view of (23) and (24), we restrict the range of $\gamma$ according to

$$
\gamma_{q} \leq \gamma<4 \gamma_{q}
$$

\section{Optimization With Respect to $\gamma$}

For a fixed value $\gamma$, the quantized coefficient vector $\mathbf{g}_{\gamma}$ can be obtained by using Procedure 4.1. The aim of this step is to obtain an optimum scaling factor $\gamma \in \Gamma$ where $\Gamma=\left[\gamma_{q}, 4 \gamma_{q}\right]$ for which the quantized coefficient vector has a minimum error with respect to either the least-square or the min-max criteria.

- For the least-square criterion: Given a value $\gamma$, the integral squared error for the quantized solution can be expressed as

$$
E\left(\mathbf{g}_{\gamma}, \gamma\right)=\gamma^{2} \mathbf{g}_{\gamma}^{T} \mathbf{Q g}_{\gamma}+\gamma \mathbf{p}^{T} \mathbf{g}_{\gamma}+\alpha .
$$

The problem becomes to minimize this error with respect to $\gamma$. This problem can be formulated as the following one dimensional optimization problem with respect to $\gamma$

$$
\min _{\gamma \in \Gamma} E\left(\mathbf{g}_{\gamma}, \gamma\right)=\min _{\gamma \in \mathcal{R}} \gamma^{2} \mathbf{g}_{\gamma}^{T} \mathbf{Q} \mathbf{g}_{\gamma}+\gamma \mathbf{p}^{T} \mathbf{g}_{\gamma}+\alpha \text {. }
$$

- For the min-max criterion: Given a value $\gamma$, the min-max error for the quantized solution can be given as

$$
F\left(\mathbf{g}_{\gamma}, \gamma\right)=\max _{\delta \in \Delta, \omega \in \Omega(\delta)} W(\omega, \delta)\left|\gamma \mathbf{g}^{T} \mathbf{s}(\omega, \delta)-H_{d}\left(e^{j \omega}, \delta\right)\right| .
$$

Consequently, the search for $\gamma$ can be formulated as the following one dimensional optimization problem:

$$
\begin{aligned}
& \min _{\gamma \in \Gamma} F\left(\mathbf{g}_{\gamma}, \gamma\right) \\
& =\min _{\gamma \in \Gamma} \max _{\delta \in \Delta} \max _{\omega \in \Omega(\delta)} W(\omega, \delta)\left|\gamma \mathbf{g}^{T} \mathbf{s}(\omega, \delta)-H_{d}\left(e^{j \omega}, \delta\right)\right| .
\end{aligned}
$$

The optimization problems (27) and (29) are nonlinear and noncontinuous optimization problems. Thus, an exhaustive search is carried out on a dense grid of $\gamma$ over the range $\Gamma$ in (25). Denote by $g_{\gamma}^{*}$ the quantized solution corresponds to the minimum cost with the corresponding scaling factor $\gamma^{*}$. This solution is referred to as the optimum scaling factor quantized (OSQ) solution. For the least-square criterion, we have

$$
E\left(\mathbf{g}_{\gamma}^{*}, \gamma^{*}\right)=\min _{\gamma \in \Gamma} E\left(\mathbf{g}_{\gamma}, \gamma\right)
$$

Similarly for the minmax solution, we have

$$
F\left(\mathbf{g}_{\gamma}^{*}, \gamma^{*}\right)=\min _{\gamma \in \Gamma} F\left(\mathbf{g}_{\gamma}, \gamma\right)
$$

$$
\max \left|\mathbf{g}_{L S, \gamma}\right|=\max _{0 \leq l \leq L-1} \max _{0 \leq n \leq N-1} \frac{\left|h_{L S, l}(n)\right|}{\gamma} \leq \max _{0 \leq l \leq L-1} \max _{0 \leq n \leq N-1} \frac{\left|h_{L S, l}(n)\right|}{4 \max _{0 \leq l \leq L-1} \max _{0 \leq n \leq N-1} \frac{\left|h_{L S, l}(n)\right|}{1-2^{-B}}}=2^{-2}-2^{-2-B}
$$




\section{DESIGN BY JOINT OPTIMIZATION FOR THE LEAST-SQUARE CRITERION}

In this section, we aim to improve further the OSQ solution $\left(\mathrm{g}_{\gamma}^{*}, \gamma^{*}\right)$ for the least-square criterion by solving the joint optimization problem of $\mathbf{g}$ and $\gamma$ with the search region reduced to around the neighborhood of the OSQ solution $\left(\mathbf{g}_{\gamma}^{*}, \gamma^{*}\right)$. That is, we do not obtain $\mathrm{g}$ from $\mathbf{h}_{L S}$ by a quantization process but rather determine the optimal discrete $\mathrm{g}$ jointly with the optimal $\gamma$. The branch and bound algorithm is employed to search for a global solution of the joint optimization problem in the reduced region. The reason that we concentrate on the least-square criterion instead of the min-max criterion is that the bound for the branch and bound algorithm can be obtained with low complexity.

Denote by $\mathbf{v}$ and $\mathbf{u}$ the lower and upper limits for the discrete coefficients $\mathrm{g}$ in the reduced region. For all $0 \leq l \leq L-1$ and $0 \leq n \leq N-1$, the values of $v_{l}(n)$ and $u_{l}(n)$ of $\mathbf{v}$ and $\mathbf{u}$ can be obtained from $g_{\gamma, l}^{*}(n)$ as follows

$$
v_{l}(n)=g_{\gamma, l}^{*}(n)-\xi \text { and } u_{l}(n)=g_{\gamma, l}^{*}(n)+\xi
$$

where $\xi$ is a positive integer. Consequently, the reduced region $\mathcal{G}^{r}$ of $\mathbf{g}$ containing $\mathbf{g}_{\gamma}^{*}$ can be defined as

$$
\begin{aligned}
& \mathcal{G}^{r}=\left\{\mathbf{g} \mid g_{l}(n) \in \mathcal{N},\right. v_{l}(n) \leq g_{l}(n) \leq u_{l}(n) \\
&\forall 0 \leq l \leq L-1,0 \leq n \leq N-1\}
\end{aligned}
$$

where $\mathcal{N}$ denotes the set of integers in $[-\infty, \infty]$.

The optimization problem in the reduced search region can be expressed as

$$
\left\{\begin{array}{l}
\min _{\mathbf{g} \in \mathcal{G}^{r}, \gamma \in \mathcal{R}} \gamma^{2} \mathbf{g}^{T} \mathbf{Q g}+\gamma \mathbf{p}^{T} \mathbf{g}+\alpha \\
\sum_{l=0}^{L-1} \sum_{n=0}^{N-1} c_{l, n} \leq M .
\end{array}\right.
$$

The basic idea of the branch and bound algorithm can be found in e.g., [16]. The problem is divided into a number of sub-problems, referred to as branches or nodes, by restricting a subset of coefficients in $\mathrm{g}$ to finite precisions. For each node, a lower bound cost of all possible discrete solutions in the branch is then obtained by formulating and solving a relaxation problem. This lower bound can then be used to decide whether to immediately cut the node or to allow further branching division. Note that while the idea of the branch and bound algorithm is generally known, the process of formulating the relaxation problem and the procedure of cutting/dividing the branches are problem dependent.

In the following, we present an efficient scheme to obtain a lower bound for each branch. For a branch, denote by $\mathbf{r}$ the set of coefficient in $\mathrm{g}$ which is discrete. For simplicity, assume that $\mathbf{r}$ is the first $k$ coefficients. The coefficient $\mathbf{h}$ of the VDF can be expressed as

$$
\mathbf{h}=\gamma \mathbf{g}=\left[\begin{array}{ll}
\gamma \mathbf{r}^{T} & \mathbf{s}^{T}
\end{array}\right]^{T}
$$

where $\mathbf{s}$ is the remainder $N L-k+1$ infinite precision coefficients.
1) Procedure 5.1: Obtain a lower bound for a branch with the discrete coefficient vector $\mathbf{r}$.

Step 1) Express the coefficient vector $\mathbf{h}$ in (35) in terms of the unknown parameters $\gamma$ and $\mathbf{s}$ as follows:

$$
\mathbf{h}=\mathbf{R}\left[\begin{array}{ll}
\gamma & \mathbf{s}^{T}
\end{array}\right]^{T}
$$

where $\mathbf{R}$ is a $N L \times N L-k+1$ matrix given by

$$
\mathbf{R}=\left[\begin{array}{cc}
\mathbf{r} & \mathbf{0}_{N L-k, N L-k} \\
\mathbf{0}_{N L-k, 1} & \mathbf{I}_{N L-k, N L-k}
\end{array}\right]
$$

and $\mathbf{I}_{N L-k, N L-k}$ is an identity matrix.

Step 2) Formulate the relaxation optimization problem with variables $\gamma$ and $\mathbf{s}$ according to the following:

$$
\min _{\gamma \in \mathcal{R}, \mathbf{s} \in \mathcal{R} N L-k}\left[\begin{array}{ll}
\gamma & \mathbf{s}^{T}
\end{array}\right] \mathbf{R}^{T} \mathbf{Q R}\left[\begin{array}{ll}
\gamma & \mathbf{s}^{T}
\end{array}\right]^{T}+\mathbf{p}^{T} \mathbf{R}\left[\begin{array}{ll}
\gamma & \mathbf{s}^{T}
\end{array}\right]^{T}+\alpha .
$$

Step 3) Obtain the infinite precision solution for (38) as

$$
\left[\gamma \mathbf{s}^{T}\right]^{T}=-\frac{1}{2} \mathbf{p}^{T} \mathbf{R}\left(\mathbf{R}^{T} \mathbf{Q R}\right)^{-1}
$$

The lower bound for the branch is the cost of the relaxation problem in (38) corresponding to the coefficient vector (39). Thus, the lower bound calculation a for each branch is relatively simple as the solution can be expressed in (39).

In the following, the lower bound for branch obtained from Procedure 5.1 will be used to decide whether to cut the branch or to allow further branching division. Let us denote the best discrete solution obtained up to this branch as $\left(\mathrm{g}^{0}, \gamma^{0}\right)$ with the corresponding cost $E\left(\mathbf{g}^{0}, \gamma^{0}\right)$.

2) Procedure 5.2: Decision on the branch or node.

Step 1) Obtain the optimum solution (39) of the relaxation problem for the branch by using Procedure 5.1.

Step 2) Obtain the quantized solution $\left[\mathbf{r}_{\gamma}, \mathbf{s}_{\gamma}\right]$ from $[\gamma \mathbf{r}, \mathbf{s}]$ with the scaling factor $\gamma$ and the constraint (34) on the total number of SPT terms using Procedure 4.1.

Step 3) If $E\left(\left[\mathbf{r}_{\gamma}, \mathbf{s}_{\gamma}\right], \gamma\right)<E\left(\mathbf{g}^{o}, \gamma^{o}\right)$, then update $\left(\mathbf{g}^{o}, \gamma^{o}\right)$ and $E\left(\mathbf{g}^{o}, \gamma^{o}\right)$.

Step 4) The current node will be removed if:

i) The current relaxation cost is greater than or equal to $E\left(\mathbf{g}^{o}, \gamma^{o}\right)$; or

ii) The total number of SPT terms in the first $k$ discrete coefficients $\mathbf{r}_{\gamma}$ is greater than the total number of SPT terms in $\mathbf{r}$ plus a positive integer $\beta$, which specifies the maximum deviation for the number of SPT terms [10]. The node can also be removed if it has a certain length and is closed to the discrete solution $\mathrm{g}^{0}$ according to the Euclidean distance measure.

Step 5) If Step 4 has not been satisfied then branching is to continue from the current branch.

In the following, we will describe the branch and bound algorithm incorporating Procedures (5.1) and (5.2).

3) Procedure 5.3: Branch and bound algorithm for optimizing (34) in the reduced region $\mathcal{G}^{r}$. 
TABLE I

VDF WiTh MIN-MAX CRITERION. MIN-MAX ERROR [DB]

\begin{tabular}{|c|c|c|c|c|}
\hline $\begin{array}{c}\text { Infinite precision } \\
\text { with least } \\
\text { square criterion }\end{array}$ & $\begin{array}{c}\text { Optimized filter } \\
\text { with 78 SPT } \\
\text { given in [13] }\end{array}$ & $\begin{array}{c}\text { OSQ solution } \\
\text { with } M=70 \text { and } \\
\text { infinite precision } \gamma\end{array}$ & $\begin{array}{c}\text { OSQ solution with } \\
M=70 \text { and quantized } \\
\gamma \Rightarrow \text { total 75 SPT }\end{array}$ & $\begin{array}{c}\text { Infinite precision } \\
\text { with min-max } \\
\text { criterion }\end{array}$ \\
\hline-42.53 & -43.27 & -45.85 & -45.95 & -47.19 \\
\hline
\end{tabular}

Step 1) Initialize the minimum solution and the cost to be the same as those of the OSQ solution, $\left(\mathrm{g}^{o}, \gamma^{o}\right)=$ $\left(\mathbf{g}_{\gamma}^{*}, \gamma^{*}\right), E\left(\mathbf{g}^{o}, \gamma^{o}\right)=E\left(\mathbf{g}_{\gamma}^{*}, \gamma^{*}\right)$ and set $k=1$.

Step 2) Let $l=\lfloor(k-1) / N\rfloor$ and $n=k-1-l N$, where $\lfloor\cdot\rfloor$ denotes the floor operator. Then, the coefficient $g_{l}(n)$ the $k^{\text {th }}$ coefficient in the vector $\mathrm{g}$. Set $\mathcal{G}^{k}$ as the set of all possible values of $g_{l}(n)$ in the reduced region

$$
\mathcal{G}^{k}=\left\{g_{l}(n) \in \mathcal{N}, v_{l}(n) \leq g_{l}(n) \leq u_{l}(n)\right\} .
$$

Since the branching is taken place according to the order of coefficients in $\mathbf{g}$, all the coefficients before $g_{l}(n)$ are fixed and discrete.

Step 3) Choose a discrete value $g \in \mathcal{G}^{k}$ and set $g_{l}(n)=g$. Reduce the discrete set $\mathcal{G}^{k}=\mathcal{G}^{k} \backslash g$ and go to Step 4.

Step 4) Denote by $\mathbf{r}=\left[g_{0}(0), \ldots, g_{l}(n)\right]^{T}$ the set of fixed discrete coefficients for the current branch. We have the following two cases.

- If $k=N L$ then optimize the scaling factor $\gamma$ corresponding to $\mathbf{r}$ that minimizes $E(\mathbf{r}, \gamma)$ and update $\left(\mathbf{g}^{o}, \gamma^{o}\right)$ only if $E(\mathbf{r}, \gamma)<E\left(\mathbf{g}^{o}, \gamma^{o}\right)$. Choose the largest index $k_{1} \leq k$ such that $\mathcal{G}^{k_{1}} \neq$ $\emptyset$. If $k_{1}$ exists then set $k=k_{1}$ and return to Step 3, otherwise go to Step 5.

- If $k<N L$ then apply Procedure 5.2 to the current branch with the discrete coefficient $\mathbf{r}$. There are two cases: (1) If the current node is removed then choose the largest index $k_{1} \leq k$ such that $\mathcal{G}^{k_{1}} \neq \emptyset$. If $k_{1}$ exists then set $k=k_{1}$ and return to Step 3, otherwise go to Step 5; (2) If branching is to continue from the current node then set $k=k+1$ and go to Step 2 .

Step 5) Stop the algorithm. The minimum solution is $\left(\mathrm{g}^{o}, \gamma^{o}\right)$ with the corresponding cost value $E\left(\mathbf{g}^{o}, \gamma^{o}\right)$.

\section{DESIGN EXAMPLES}

1) Example 1: Consider the design of a discrete VDF with infinite precision coefficients provided in [6] and min-max criterion [12], [13]. The bandwidth under consideration for the filter is from 0 to $0.6 \pi$. The length of each FIR filters used in the Farrow structure is $N=8$ and $L=4$. The number of bits is $b=10$ and the range for $\delta$ is chosen as $\Delta=[-0.5,0.5]$.

Table I shows the maximum error deviation $20 \log _{10} F(\mathbf{h})$ [dB] for: (i) the infinite precision solution with least-square criterion, (ii) the optimized sum of SPT coefficient solution given in [13], (iii) the OSQ solution with $M=70$ and infinite precision $\gamma$ obtained according to Section IV with 2000 points, (iv) the OSQ solution with quantized $\gamma$, and (v) the infinite
TABLE II

VDF WITH MIN-MAX CRITERION. MINMAX ERROR [DB] FOR OSQ SOLUTIONS

\begin{tabular}{|c|c|c|c|c|}
\hline$\gamma$ & $M=60$ & $M=65$ & $M=70$ & $M=80$ \\
\hline Infinite precision & -44.69 & -45.41 & -45.85 & -46.68 \\
Quantized & -44.20 & -45.11 & -45.95 & -46.34 \\
\hline
\end{tabular}

precision with min-max criterion. Note that the filter (see [12, Table I]) has 78 total SPT instead of just 48 as reported (see [12, Table II]). The OSQ solution with a total of 75 SPT after taking into account 5 SPT representation for $\gamma$ has approximately the same min-max error as the OSQ with infinite precision $\gamma$. From the table, the OSQ with quantized $\gamma$ has approximately $2.7 \mathrm{~dB}$ improvement in the min-max error when compared with the optimized solution in [12] while having approximately the same the sum of SPT.

Table II shows the min-max error for the OSQ solution with infinite precision $\gamma$ and quantized $\gamma$ when $M$ increases from 60 to 80 . The OSQ solution with quantized $\gamma$ has approximately the same min-max error as the filter with infinite precision $\gamma$. For $M=60$, the filter obtained has less min-max error than the solution in [12] with fewer total SPT. Also, the min-max error reduces when $M$ increases. In particular, when $M=80$ the min-max error for the OSQ solution approaches the error for infinite precision min-max solution given in Table I. Note that these finite precision filters can also be implemented using the approach in [18].

2) Example 2: Consider the design of a bandpass VDF with variable delay and least-square criterion. The range for the tuning parameter is chosen different from Example 1, e.g., $\Delta=$ $[0,1]$. The desired group delay of the filter changes linearly according to $\delta$ as $\tau(\delta)=\tau_{d}+2 \delta$ with $\tau_{d}=9$. The desired passband and stopband regions $\mathcal{P}(\delta)$ and $\mathcal{S}(\delta)$ do not depend on the tuning parameter $\delta, \mathcal{P}(\delta)=[0.4 \pi, 0.6 \pi]$, and $\mathcal{S}(\delta)=$ $[0,0.25 \pi] \cup[0.75 \pi, \pi]$. The VDF has 4 sub-filters, $L=4$, with the length of each sub-filter chosen as $N=21$. The integer $\xi$ for the reduced search region in (32) is chosen as $\xi=2$ while the value of $\beta$ in Procedure 5.3 chosen as $\beta=2$ if $k \leq L N / 2$ and 1 otherwise. The number of allowable bits is $B=9$.

Table III shows the performance criterion $10 \log _{10} E(\mathrm{~g}, \gamma)$ [dB] for different values of $M$. The first column of the table shows the value of $M$, obtained based on the total number of VDF coefficients $N L$ as $M=\mathcal{I}\{\mu N L\}$ where $\mu$ is a real positive number increasing from 1.0 to 2.1 and $\mathcal{I}\{\cdot\}$ denotes the integer closest to real value inside $\{\cdot\}$. The second column in Table III shows the initial quantized solution obtained by using Procedure 4.1 with the scaling factor $\gamma=\gamma_{q}$. The third column presents the OSQ solution, obtained by searching for the best value of $\gamma$ over a dense grid of the search interval given by (25). The integral squared error is significantly reduced over the initial scaling factor $\gamma=\gamma_{q}$ by searching for an optimum scaling factor. The fourth column in the table shows the integral squared 
TABLE III

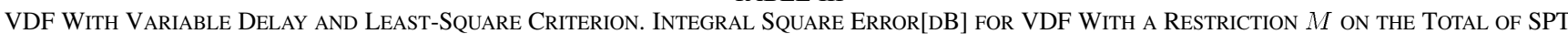
TERMS AND THE INFINITE PRECISION SOLUTION.

\begin{tabular}{|c|c|c|c|c|}
\hline $\begin{array}{c}\text { Constraint } M \\
\text { on the total } \\
\text { SPT }\end{array}$ & $\begin{array}{c}\text { Initial } \\
\text { quantized } \\
\text { solution }\end{array}$ & $\begin{array}{c}\text { Optimum scaling } \\
\text { quantized } \\
\text { solution }\end{array}$ & $\begin{array}{c}\text { Optimum solution } \\
\text { in the reduced } \\
\text { region }\end{array}$ & $\begin{array}{c}\text { Infinite } \\
\text { precision } \\
\text { solution }\end{array}$ \\
\cline { 1 - 4 } $\mathcal{I}\{1.0 N L\}=84$ & -10.99 & -18.00 & -23.31 & \\
\cline { 1 - 4 } $\mathcal{I}\{1.3 N L\}=109$ & -15.24 & -22.36 & -23.07 & \multirow{2}{*}{-32.49} \\
\cline { 1 - 4 } $\mathcal{I}\{1.6 N L\}=134$ & -22.38 & -27.49 & -29.04 & \\
\cline { 1 - 3 }$\{1.9 N L\}=160$ & -27.50 & -30.52 & -30.95 & \\
\cline { 1 - 3 } $\mathcal{I}\{2.1 N L\}=176$ & -29.71 & -31.24 & -31.80 & \\
\hline
\end{tabular}

TABLE IV

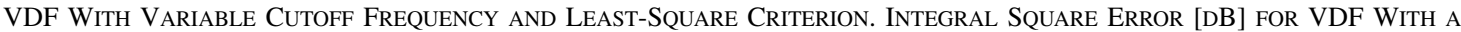
RESTRICTION $M$ ON THE TOTAL OF SPT TERMS AND the INFINITE PRECISION SOlUtion.

\begin{tabular}{|c|c|c|c|c|}
\hline $\begin{array}{c}\text { Constraint } M \\
\text { on the total } \\
\text { SPT }\end{array}$ & $\begin{array}{c}\text { Initial } \\
\text { quantized } \\
\text { solution }\end{array}$ & $\begin{array}{c}\text { Optimum scaling } \\
\text { quantized } \\
\text { solution }\end{array}$ & $\begin{array}{c}\text { Optimum solution } \\
\text { in the reduced } \\
\text { region }\end{array}$ & $\begin{array}{c}\text { Infinite } \\
\text { precision } \\
\text { solution }\end{array}$ \\
\cline { 1 - 4 } $\mathcal{I}\{1.0 N L\}=84$ & -18.11 & -24.51 & -24.51 & \\
\cline { 1 - 3 }$\{1.3 N L\}=109$ & -26.65 & -27.81 & -30.01 & \multirow{2}{*}{-35.04} \\
\cline { 1 - 4 } $\mathcal{I}\{1.6 N L\}=134$ & -28.21 & -33.13 & -33.36 & \\
\cline { 1 - 3 } $\mathcal{I}\{1.9 N L\}=160$ & -31.21 & -34.55 & -34.80 & \\
\cline { 1 - 3 }
\end{tabular}

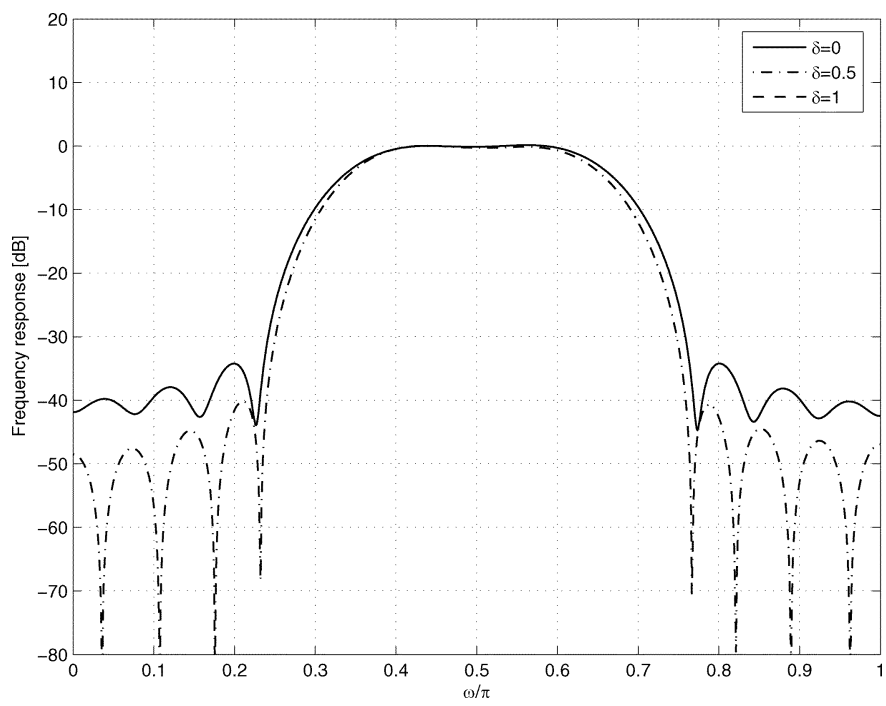

Fig. 2. VDF with variable delay and least-square criterion. Frequency response for the infinite precision solution VDF with least-square criterion.

error obtained by using the branch and bound algorithm, presented in Procedure 5.3, over a reduced region containing $\mathbf{g}_{\gamma}^{*}$. The error reduces further by jointly optimized the discrete coefficients and the scaling factor. Finally, the last column shows the integral squared error for the infinite precision solution with the least-square criterion. For $\gamma=2.1 N L$, the integral square error for the optimum discrete solution is within from $0.7 \mathrm{~dB}$ the infinite precision solution. Thus, the discrete VDF can be implemented efficiently with an average of 2.1 multipliers per coefficients. The operations of the filter coefficients in this case are reduced to simple additions and subtractions.

Figs. 2 and 3 show the magnitude responses and the group delays of the VDFs with infinite precision coefficients. The values of $\delta$ are taken as $0,0.5$ and 1 . The group delays for the three bandpass filters vary around the desired group delays, that is around 9 for $\delta=0$ and 11 for $\delta=1$. The magnitude responses of the three filters, on the other hand, have approximately the same passband and stopband cutoff frequencies.

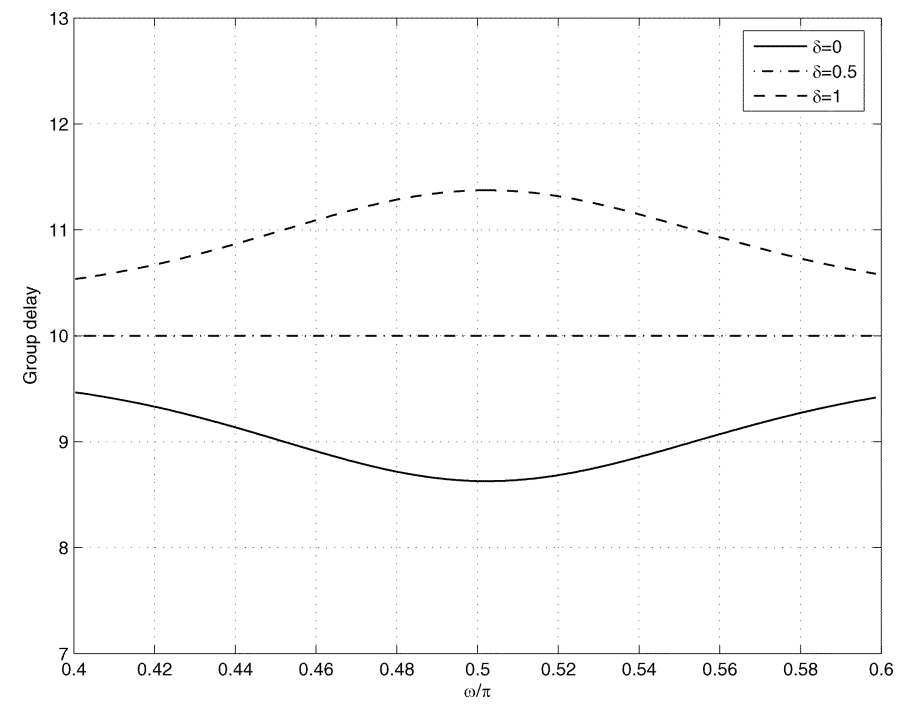

Fig. 3. VDF with variable delay and least-square criterion. Group delay for the infinite precision solution VDF with least-square criterion.

Figs. 4 and 5 plot the magnitude responses and the group delays for the optimum discrete VDFs with the sum of SPT coefficients and an upper bound $M=\mathcal{I}\{2.1 N L\}$ on the total number of SPT. The filters have the desired group delay increases around 9 to 11 with approximately the same passband and stopband regions. In addition, the discrete VDFs have approximately the same performance as the infinite precision filters.

3) Example 3: Consider the design of a bandpass VDF with variable cutoff frequencies and least-square criterion. The passband and stopband regions $\mathcal{P}(\delta)$ and $\mathcal{S}(\delta)$ change depending on the tuning parameter $\delta$ as $\mathcal{P}(\delta)=[0.3 \pi+0.2 \pi \delta, 0.5 \pi+0.2 \pi \delta]$ and $\mathcal{S}(\delta)=[0,0.15 \pi+0.2 \pi \delta] \cup[0.65 \pi+0.2 \pi \delta, \pi]$ with the range for $\delta$ chosen the same as in Example 2. The desired group delay for the filters is constant for all $\delta, \tau(\delta)=10$. Here, the integer $\xi$ for the reduced search region in (32) is chosen as $\xi=1$.

Table IV shows the integral squared error for the initial quantized solution, OSQ solution and the optimum solution in the 
TABLE V

VDF With VARiable Cutoff Frequency and Least-SQuare CRiterion. THE Number of BRANCHES OR NODES VISITED FOR THE BRANCH AND BOUND ALGORITHM

\begin{tabular}{|c|c|c|}
\hline Constraint $M$ & $\begin{array}{l}\text { The number of branches or nodes visited } \\
\text { by the branch and bound algorithm }\end{array}$ & $\begin{array}{l}\text { The total number of nodes } \\
\text { for the discrete opt. problem }\end{array}$ \\
\hline $\mathcal{I}\{1.0 N L\}=84$ & 90 & \multirow{4}{*}{$3^{84} \approx 1.197 \times 10^{40}$} \\
\hline $\mathcal{I}\{1.3 N L\}=109$ & 18279 & \\
\hline $\mathcal{I}\{1.6 N L\}=134$ & 1194 & \\
\hline $\mathcal{I}\{1.9 N L\}=160$ & 416293 & \\
\hline
\end{tabular}

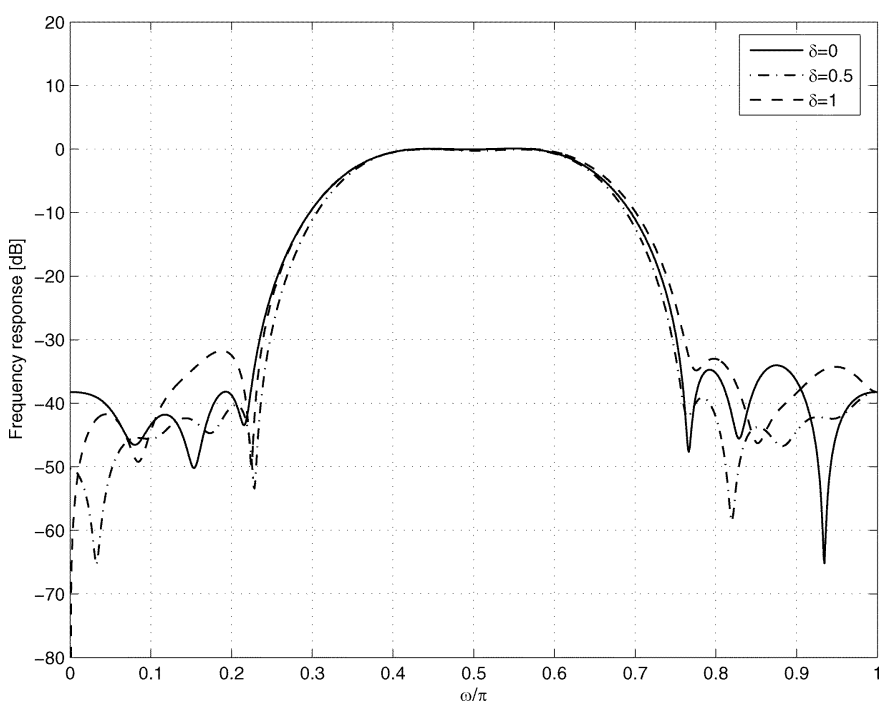

Fig. 4. VDF with variable delay and least-square criterion. Frequency response for the optimum discrete VDF with $\mu=2.1$ or $M=176$.

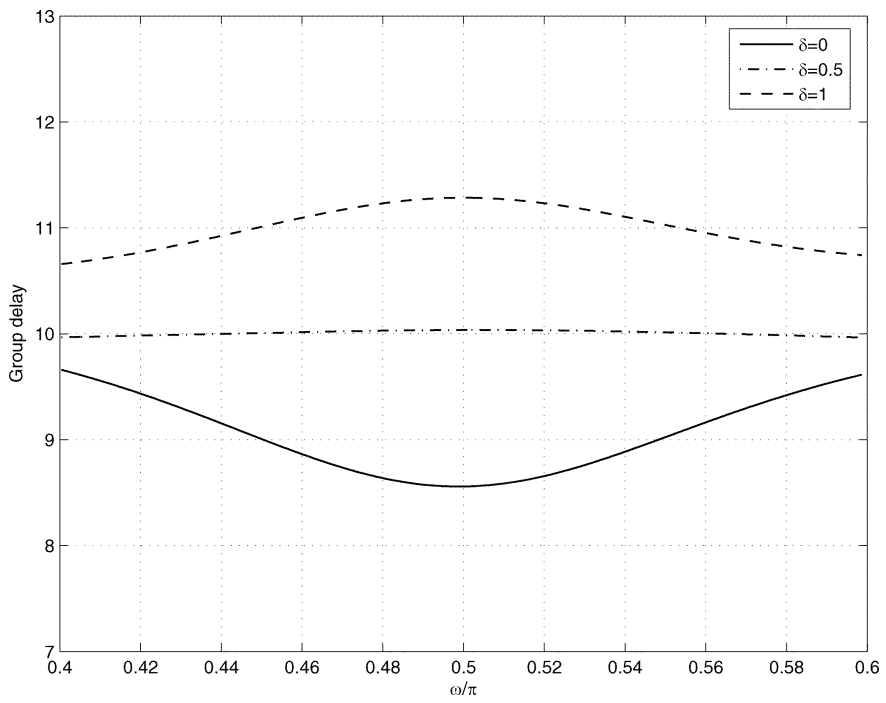

Fig. 5. VDF with variable delay and least-square criterion. Group delay for the optimum discrete VDF with $\mu=2.1$ or $M=176$.

reduced region. The error is reduced by searching for the optimum scaling factor. It is then reduced further by searching for the optimum solution in the reduced region.

Table $\mathrm{V}$ shows the total number of branches or nodes visited by the branch and bound algorithm. The third column shows the total number of possible nodes for the discrete optimization problem. Since $\xi=1$, the number of possible nodes for the problem becomes $3^{N L}=3^{84} \approx 1.197 \times 10^{40}$. It can be seen from the table that the number of visited nodes is smallest for

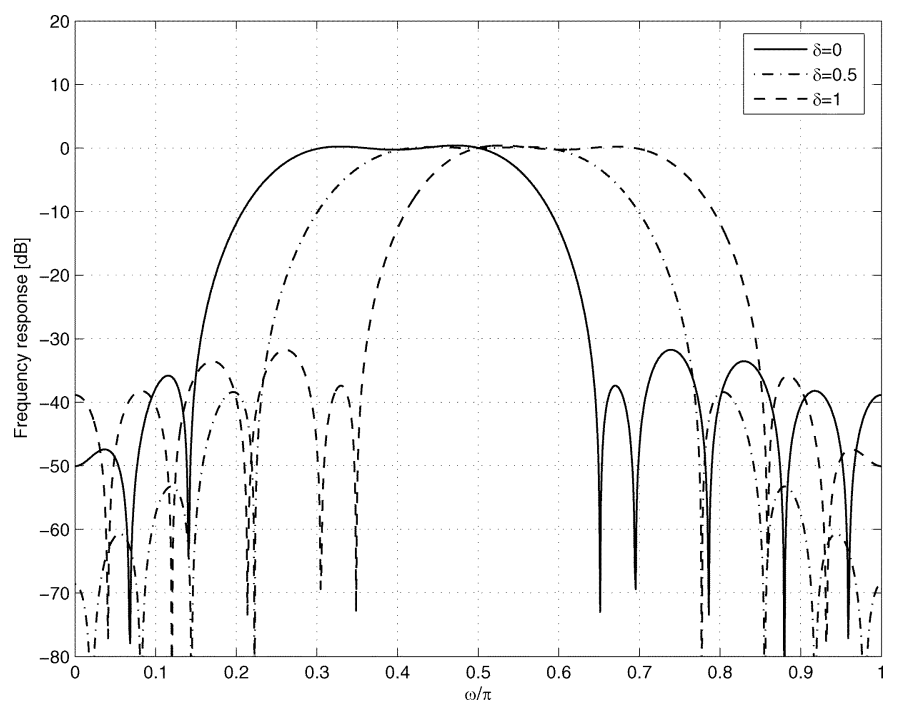

Fig. 6. VDF with variable cutoff frequency and least-square criterion. Frequency response for the infinite precision solution VDF with least-square criterion.

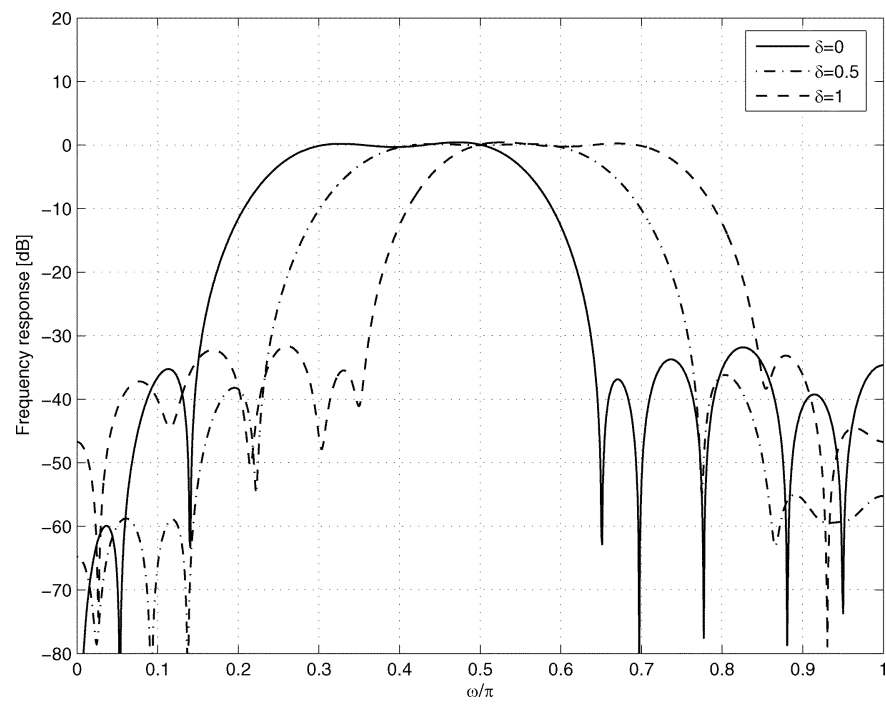

Fig. 7. VDF with variable cutoff frequency and least-square criterion. Frequency response for the near-optimum discrete VDF with $\mu=1.9$ or $M=$ 160 .

$\mu=1.0$ or 1.6 since the OQS in those cases are relatively closed to the minimum solution in the reduced region. Moreover the number of nodes visited by the algorithm is much smaller than the total number possible nodes for the discrete optimization problem.

Fig. 6 shows the frequency responses of the infinite precision VDFs with $\delta$ taken as $0,0.5$ and 1 . The frequency responses of the optimum discrete VDFs with the upper bound 
$M=\mathcal{I}\{1.9 N L\}$ on the total number of SPT terms is given in Fig. 7. This filter can be implemented with an average of only 1.9 SPT per filter coefficient with approximately the same performance as the infinite precision solution.

\section{CONCLUSION}

In this paper, a two-step and a three-step schemes are proposed for the design of VDFs with the sum of SPT coefficients and minmax or least-square criterion. For the least-square criterion, the effective application of the branch and bound method for solving this complex nonlinear integer programming problem has been possible through the introduction a reduced search area and an efficient cutting scheme. Design example shows a significant improvement by using the proposed method over merely quantizing the infinite precision solution. In addition, the numerical examples presented show that the finite precision filters can obtained approximately the same performance as the infinite precision solution with a small number of additions and subtractions.

\section{REFERENCES}

[1] X. Li, H. Zhao, M. Li, and J. Wu, "A design for variable fractional delay FIR filters," in Proc. Int. Conf. Commun., Circuits Syst., Jun. 2004, vol. 2, pp. 1131-1135.

[2] H. Johansson and P. Lowenborg, "On the design of adjustable fractional delay FIR filters," IEEE Trans. Circuits Syst. II, Analog Digit. Signal Process., vol. 50, no. 4, pp. 164-169, Apr. 2003.

[3] T. B. Deng, "Discretization-free design of variable fractional-delay FIR digital filters," IEEE Trans. Circuits Syst. II, Analog Digit. Signal Process., vol. 48, no. 6, pp. 637-644, Jun. 2001.

[4] T. I. Laakso, V. Valimaki, M. Karjalainen, and U. K. Laine, "Splitting the unit delay: Tools for fractional delay filter design," IEEE Signal Process. Mag., vol. 13, no. 1, pp. 30-60, Jan. 1996.

[5] K. Khamei, A. Nabavi, and S. Hessabi, "Design of variable fractional delay FIR filters using genetic algorithm," in Proc. IEEE Int. Conf. Electron., Circuits Syst., Dec. 2003, vol. 1, pp. 48-51.

[6] C. W. Farrow, "A continuously variable digital delay element," in Proc. IEEE Int. Symp. Circuits Syst., Jun. 1988, vol. 3, pp. 2641-2645.

[7] C.-L. Chen and A. N. Willson, "A trellis search algorithm for the design of FIR filters with signed-powers-of-two coefficients," IEEE Trans. Circuits Syst. II, Analog Digit. Signal Process., vol. 46, no. 1, pp. 29-39, Jan. 1999.

[8] H. H. Dam, S. Nordebo, K. L. Teo, and A. Cantoni, "FIR filter design over discrete coefficients and least-square error," Proc. IEE Vision, Image Signal Process., vol. 147, no. 6, pp. 543-548, Dec. 2000.

[9] J.-J. Shyu and Y.-C. Lin, "A new approach to the design of discrete coefficient FIR digital filters," IEEE Trans. Acoust., Speech, Signal Process., vol. 43, no. 1, pp. 310-314, Jan. 1995.

[10] W. R. Lee, L. Caccetta, K. L. Teo, and V. Rehbock, "A branch and bound based approach to digital filter design with power-of-two coefficients," in Proc. ICOTA, Dec. 2004, ISBN: 18768115, CDROM.

[11] Y. C. Lim, "Design of discrete-coefficient-value linear phase FIR filters with optimum normalized peak ripple magnitude," IEEE Trans. Circuits Syst., vol. 37, no. 12, pp. 1480-1486, Dec. 1990.

[12] C. K. S. Pun, Y. C. Wu, S. C. Chan, and K. L. Ho, "On the design and efficient implementation of the Farrow structure," IEEE Signal Process. Lett., vol. 10, no. 7, pp. 189-192, Jul. 2003.

[13] C. K. S. Pun, S. C. Chan, K. S. Yeung, and K. L. Ho, "On the design and implementation of FIR and IIR digital filters with variable frequency charactericstics," IEEE Trans. Circuits Syst. II, Analog Digit. Signal Process., vol. 49, no. 11, pp. 689-703, Nov. 2002.

[14] J. Y. Kaakinen and T. Saramaki, "Multiplier-free polynomial-based FIR filters with an adjustable fractional delay," in Proc. 9th Int. Conf. Syst., Sep. 2002, vol. 3, pp. 1167-1170.

[15] D. Li, Y. C. Lim, Y. Lian, and J. Song, "A polynomial time algorithm for designing FIR filters with power-of-two coefficients," IEEE Trans. Signal Process., vol. 50, no. 8, pp. 1935-1941, Aug. 2002.

[16] Optimisation, G. L. Nemhauser, A. H. G. R. Kan, and M. J. Todd, Eds. Amsterdam, The Netherlands: North-Holland, 1989.
[17] H. H. Dam, A. Cantoni, K. L. Teo, and S. Nordholm, "Variable digital filter with least-square criterion and peak gain constraints," IEEE Trans. Circuits Syst. II, Express Briefs, vol. 54, no. 1, pp. 24-28, Jan. 2007.

[18] A. G. Dempster and M. D. Macleod, "Use of minimum-adder multiplier blocks in FIR digital filters," IEEE Trans. Circuits Syst. II, Analog Digit. Signal Process., vol. 42, no. 9, pp. 569-577, Sep. 1995.

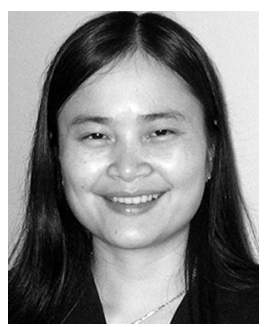

Hai Huyen Dam received the Bachelor's (first class Honors) and Ph.D. degrees (with distinction) from Curtin University of Technology, Perth, WA, Australia in 1996 and 2001, respectively.

From 1999 to 2000, she spent one year at the Blekinge Institute of Technology, Ronneby, Sweden as a Visiting Researcher. From 2001 to 2005, she was a Research Fellow and Senior Research Fellow with Western Telecommunications Research Institute (WATRI), Curtin University of Technology. Since 2006, she is a Senior Lecturer with a joint appointment between WATRI and the Department of Mathematics and Statistics, Curtin University of Technology. Her research interests are adaptive array processing, optimization methods, equalization, and filter design.

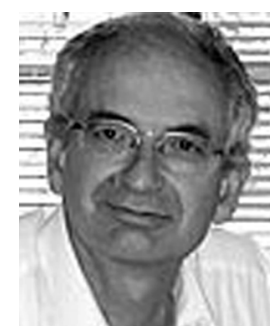

Antonio Cantoni (M'74-SM'83-F'98) was born in Soliera, Italy, on 30 October, 1946. He received the B.E. (first class honors) and Ph.D. degrees from the University of Western Australia, Nedlands, WA, Australia, in 1968 and 1972, respectively.

He was a Lecturer in Computer Science at the Australian National University, Canberra, Australia, in 1972. He joined the Department of Electrical and Electronic Engineering at the University of Newcastle, Shortland, NSW, Australia, in 1973, where he held the Chair of Computer Engineering until 1986. In 1987, he joined QPSX Communications Ltd, Perth, WA, Australia, as Director of the Digital and Computer Systems Design Section for the development of the DQDB Metropolitan Area Network. From 1987 to 1990, he was also a Visiting Professor in the Department of Electrical and Electronic Engineering at the University of Western Australia, Nedlands, WA, Australia. From 1992 to 1997, he was the Director of the Western Australian Telecommunications Research Institute and Professor of Telecommunications at Curtin University of Technology, Perth, WA, Australia. During this period, he was also the Director of the Cooperative Research Centre for Broad-Band Telecommunications and Networking. He is currently Chief Technology Officer with Atmosphere Networks Inc., and Professor of Telecommunications at the University of Western Australia. He is interested in adaptive signal processing, electronic system design, and networking and regularly acts as a Consultant to industry in these areas.

Dr Cantoni is a Fellow of the Australian Academy of Technological Sciences and Engineering. He has been an Associate Editor of the IEEE TRANSACTIONS ON SIGNAL PROCESSING.

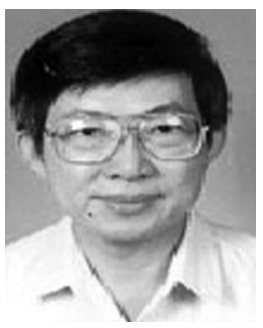

Kok Lay Teo (M'74-SM'87) received the B.Sc. degree in telecommunications engineering from Ngee Ann Technical College, Singapore, and the M.A.Sc. and $\mathrm{Ph} . \mathrm{D}$. degrees in electrical engineering from the University of Ottawa, ON, Canada.

He was with the Department of Applied Mathematics, University of New South Wales, Sydney, NSW, Australia, the Department of Industrial and Systems Engineering, National University of Singapore, Singapore, the Department of Mathematics, the University of Western Australia, Nedlands, WA, Australia. In 1996, he joined the Department of Mathematics and Statistics, Curtin University of Technology, Perth, WA, Australia, as Professor. He then took up the position of Chair Professor of Applied Mathematics and Head of Department of Applied Mathematics at the Hong Kong Polytechnic University, Hong Kong, from 1999 to 2004. He is currently Chair of Applied Mathematics and Head of Department of Mathematics and Statistics at Curtin University of Technology. His research interests include both the theoretical and practical aspects of optimal control and optimization, and their practical applications such as in signal processing in telecommunications, and financial portfolio 
optimization. He has published 5 books and over 300 journal papers. He has a software package, MISER3.3, for solving general constrained optimal control problems. He is Editor-in-Chief of the Journal of Industrial and Management Optimization, and serves as an Associate Editor of a number of international journals, including Automatica, Nonlinear Dynamics and Systems Theory, Journal of Global Optimization, Engineering and Optimization, and Discrete and Continuous Dynamic Systems (Series A and Series B), Dynamics of Continuous, Discrete and Impulsive Systems (Series A and Series B).

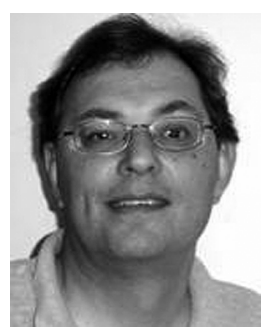

Sven Nordholm (M'91-SM'04) was born in Tollarp, Sweden, in 1960. He received the M.Sc.EE degree in civil engineering, the Licentiate of engineering degree, and the $\mathrm{Ph} . \mathrm{D}$. degree in signal processing from Lund University, Lund, Sweden in 1983, 1989, and 1992, respectively.

He was one of the Founders of the Department of Signal Processing at the Blekinge Insitute of Technology (BTH), Ronneby, Sweden. At BTH, he held positions as Lecturer, Senior Lecturer, Associate Professor, and Professor. Since 1999, he as been at the Curtin University of Technology, Perth, WA, Australia. From 1999-2002 he was a Director of the Australian Telecommunications Research Institute (ATRI), Perth, WA, Australia, and a Professor at Curtin University of Technology. He is currently Professor and Director, Signal Processing Laboratories at Western ATRI, a joint institute between the University of Western Australia and Curtin University of Technology. He is also Chief Technological Officer and Co-Founder of a start-up company Sensear. His main research efforts have been spent in the fields of speech enhancement, adaptive and optimum microphone arrays, acoustic essay canellation, adaptive signal processing, sub-band adaptive filtering, and filter design. 